\title{
Opioid addiction treatment
}

\section{Commentary}

Our brains have opioid receptors (receptacles) that become activated when opioid drugs (plugs) dock into the receptor sites. Once the connection is made between the receptor and an opioid drug, an electrical impulse is sent down a series of nerve pathways to specific areas in our brain causing the opioid effect (euphoria, analgesia, stress relief). Current research in drug treatment is aimed at manipulating the receptor sites with drugs that "trick our brain receptors to stabilize" thus reducing the withdrawal symptoms and cravings that make it so difficult for people to stop using mood altering drugs. Once the brain is stabilized, a patient can then start behavioral therapy to alter the behaviors acquired while they were actively dependent on their opioid of abuse.

Currently there are two FDA approved drugs for replacement therapy including Methadone and Buprenorphine. Methadone has been in use for a number of years while Buprenorphine is a relatively new drug (approved for use in 2002). Both drugs work by stabilizing the opioid receptor in the brain. Methadone is a pure opioid receptor agonist meaning it is a perfect fit (plug) into the receptor (receptacle) causing complete (1 to 1 ) stimulation of the receptor. Buprenorphine is a partial agonist antagonist meaning it causes an imperfect fit causing incomplete (limited) stimulation of the receptor. Though both drugs effectively eliminate withdrawal symptoms and cravings, Buprenorphine is much safer because you can only stimulate the receptor to a certain point before achieving a plateau effect (no further effect with increasing the dose). The 1 to 1 stimulating effect of Methadone causes a much greater risk of potential complications, including respiratory and circulatory collapse. Other clinically significant advantages of Buprenorphine, in addition to its safety profile, include:

a. No tolerance effect, thus reducing the need for increasing doses to achieve the desired result.

b. No euphoric effect or cognition changes (no altered consciousness and/or diminished mental acuity).

c. The antagonist (blocking) effect of Buprenorphine makes it virtually impossible for other opioids to attach to the opioid receptor sites, thus blocking any reinforcing effect of the opioid of abuse.

d. Buprenorphine can be prescribed by a qualified MD in his/ her office without the need for patients to travel to a Federally designated Methadone clinic and Buprenorphine can be prescribed for periods of 30 to 60 days, unlike Methadone which generally requires daily visits to the clinic for administration of a single daily dose.

e. Diversion of Buprenorphine to the "street" is much lower compared to Methadone.

Any successful opioid treatment plan must be tailored to the individual needs of each patient. Many patients have co-morbid conditions (depression, Bipolar disorder, general anxiety disorders, etc) that must be treated separately from their primary addiction.
Volume 3 Issue 4 - 2017

\author{
Rick Campana \\ Diplomate of American Board of Addiction Medicine, USA
}

Correspondence: Rick Campana, Diplomate of American Board of Addiction Medicine, Eastern Virginia Medical School, Norfolk,Virginia, USA, Email rickcampana I@hotmail.com

Received: May 12, 2017 | Published: May 22, 2017

Failure to properly identify and treat a patient's co-morbid conditions will significantly reduce their chance of prolonged recovery from their opioid dependency. Patients must feel comfortable in their treatment setting and not be made to feel like they are lepers or rejects of society. When I initially meet my opioid dependent patients, I tell them to leave their shame and guilt at my front door. I advise them that they have a genetically inherited brain disease influenced by their social environment and that I view them on the same level as my medical patients (diabetics, hypertensives, heart disease, etc). Once a patient understands the bio-social aspects of their addiction, the vast majority of them vigorously embrace treatment and end up doing quite well. I have found that opioid dependent patients require a minimum of six months of Buprenorphine treatment in order for them to achieve meaningful results with their behavioral therapy. Anything short of this time period usually results in relapse. Finally, I strongly believe that general wellness must be integrated into any standard opiate treatment program including regular exercise, diet and stress management. I have discovered that many opioid dependent patients smoke cigarettes and live unhealthy life styles. Those patients who embrace a healthy life style typically do much better with their recovery. As a side note, I have had much success using the new smoking cessation drug called Chantix for helping my patients stop smoking. Chantix has properties similar to Buprenorphine and is very effective in stabilizing the nicotine brain receptors preventing withdrawal symptoms and cravings while completely blocking the nicotine receptors, thus eliminating positive reinforcing effects from smoking.

In summary, optimal treatment for opiate addiction should include an individualized tailored program that incorporates:

a. Buprenorphine to stabilize the brain (controlling withdrawal symptoms, cravings and to block any reinforcing effects of the abused opioid drug).

b. Evaluation and treatment for any co-morbid conditions by a qualified mental health professional.

c. Behavioural therapy to address triggers, stress management and coping skills.

d. A wellness program emphasizing healthy lifestyle choices. 


\section{Acknowledgements}

None.

\section{Conflict of interest}

The author declares no conflict of interest. 